\title{
Prospects for a DVCS measurement at COMPASS
}

\author{
Eva-Maria Kabuß (for the COMPASS Collaboration) ${ }^{* \dagger}$ \\ Institut für Kernphysik, Mainz University, D 55099 Mainz. \\ E-mail: emk@kph.uni-mainz.de
}

The high energy polarised muon beam available at CERN with the option of using positive or negative muons with opposite polarisation gives COMPASS an excellent possibility to study generalised parton distributions via deeply virtual Compton scattering. In a first step we propose to use an unpolarised proton target to study the slope of the $t$ dependence of the differential cross section as a function of $x_{B j}$ to observe a possible shrinkage of the nucleon with increasing $x_{B j}$. Furthermore, the beam charge and spin difference will be measured over a wide kinematical range to determine the Compton form factor related to the GPD $H$. As a second step we consider to use a transversely polarised proton target to collect data to constrain the GPD $E$. In preparation of the future measurements two DVCS test runs were performed in 2008 and 2009.

XVIII International Workshop on Deep-Inelastic Scattering and Related Subjects, DIS 2010 April 19-23, 2010

Firenze, Italy

\footnotetext{
*Speaker.

† supported by the BMBF
} 


\section{Introduction}

During the recent years the framework of generalised parton distributions (GPDs) was developed $[1,2,3]$ providing a comprehensive description of the partonic structure of the nucleon and containing a wealth of new information. It embodies both, form factors as observed i.e. in elastic electron scattering and parton distribution functions (PDFs) measured in deep inelastic lepton scattering. A GPD can be considered as a momentum-dissected form factor providing information on the transverse localisation of a parton as a function of the fraction it carries of the nucleon's longitudinal momentum. Thus one obtains a "3-dimensional picture" of the nucleon which is often referred to as "nucleon tomography" [4]. GPDs also allow access to such a fundamental property of the nucleon as the orbital angular momentum of quarks [3].

GPDs are experimentally accessible in exclusive reactions such as deeply virtual Compton scattering (DVCS) and deeply virtual meson production (DVMP). In the COMPASS kinematic domain the GPD $H$ yields the dominant contribution in measurements with an unpolarised target, while the GPDs $H$ and $E$ are accessible with a transversely polarised target. They depend on the photon virtuality $Q^{2}$, the total four-momentum transfer squared $t$ between the initial and final nucleon states and two additional variables $x$ and $\xi$ representing the average and half the difference between the initial and final longitudinal momentum fraction of the nucleon carried by the parton (see Fig.1 left). In DVCS and DVMP processes $x$ is an internal variable while the skewness $\xi$ is related to the Bjorken variable $x_{B j}=Q^{2} / 2 M v$ in the Bjorken limit: $\xi=x_{B j} /\left(2-x_{B j}\right)$.

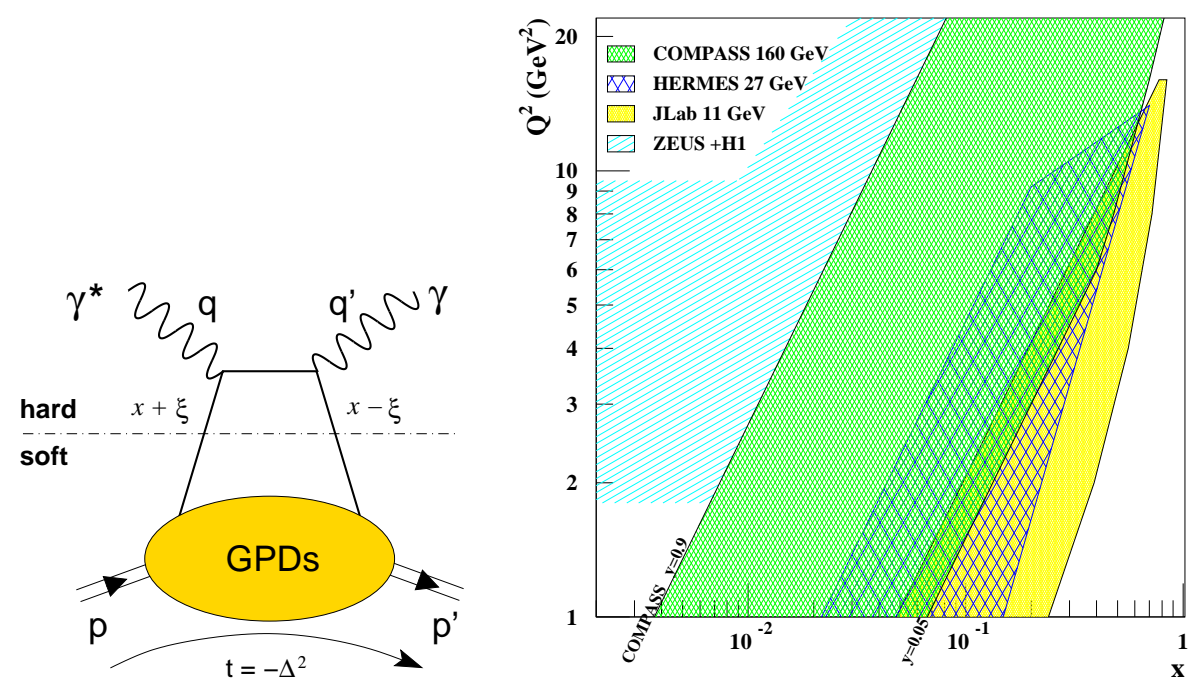

Figure 1: Left: Handbag diagram for the DVCS process at leading twist. Right: Kinematic domains for measurements of hard exclusive processes.

The extraction of GPDs from such measurements needs experimental data on hard exclusive processes in a broad kinematic range. Up to now information in the high energy regime at low $x_{B j}$ was provided by H1 and ZEUS at DESY and by HERMES and JLAB experiments in the low energy regime at high $x_{B j}$. Missing are high precision data in the kinematic regime where both sea and valence quarks are equally important. 


\section{Proposed set-up}

A DVCS measurement was recently proposed by the COMPASS collaboration [5] at the high energy muon beam of the CERN SPS. It is forseen in two phases, the first one in the present proposal uses a liquid hydrogen target to extract the GPD $H$. A second phase with a polarised target will be presented in an addendum to the present proposal in the future. Data on exclusive vector meson production will be recorded simultaneously to the DVCS measurement.

At present the COMPASS experiment [6] consists of a two stage spectrometer, each stage equipped with tracking detectors, electromagnetic and hadron calorimeters and PID detectors. For the first part of the DVCS measurements a $2.5 \mathrm{~m}$ long liquid hydrogen target will be used. Installing a $4 \mathrm{~m}$ long recoil proton detector around the target will ensure exclusivity of the DVCS and DVMP events. A prototype of a quadrant of this detector was already tested in the muon beam. For low values of $x_{B j}$ the photons are detected in the existing electromagnetic calorimeters, while for higher values of $x_{B j}$ an additional large angular acceptance calorimeter ECAL0 has to be added. The technical solution for this calorimeter is presently under study. With such a set-up a region of $0.005<x_{B j}<0.27$ is accessible as shown in Fig.1 right. Data will be collected with $\mu^{+}$and $\mu^{-}$ beams. Assuming 2 years of data taking (equivalent to an integrated luminosity of $1222 \mathrm{pb}^{-1}$ ) at a global efficieny of $10 \%$ reasonable statistics can be accumulated up to $Q^{2}$ values of $8(\mathrm{GeV} / c)^{2}$.

To obtain insight into the GPD $E$ and to study the role of orbital angular momentum of quarks, transverse target spin asymmetries for DVCS are important observables. They will be accessed in the second part of the program using a transversely polarised ammonia target similar to the one presently used by COMPASS. In this case the recoil detector will have to be integrated into the cryostat of the superconducting solenoid. As an alternative solution the use of an externally polarised target with only a small holding field is studied. In this approach a more conventional recoil detector can be used.

\section{Proposed measurements}

The main objective of the proposal is a measurement of DVCS as it is considered the theoretically cleanest of the experimentally accessible processes because effects of next-to-leading order and subleading twists are under theoretical control [7]. A competing process is the Bethe-Heitler (BH) process of elastic lepton-nucleon scattering with a hard Bremsstrahlung photon emitted by the incoming or outgoing lepton. It produces the same final state as DVCS so that both processes interfere at the level of amplitudes. In the COMPASS experiment, kinematic domains are accessible where either BH or DVCS dominate. At low $x_{B j}$ almost pure BH events are recorded which can be used as an excellent reference yield. The collection of almost pure DVCS events at higher $x_{B j}$ will allow a measurement of the $t$ dependence of the cross section which is related to the tomographic image of the nucleon. In the intermediate domain the DVCS contribution will be enhanced by the $\mathrm{BH}$ process through their interference.

The differential cross section for muon production of real photons on an unpolarised proton target is given by

$$
\frac{d^{4} \sigma(\mu p \rightarrow \mu p \gamma)}{d x_{B j} d Q^{2} d|t| d \phi}=d \sigma^{B H}+\left[d \sigma_{\text {unpol }}^{D V C S}+P_{\mu} d \sigma_{\text {pol }}^{D V C S}\right]+e_{\mu}\left[\operatorname{ReI}+P_{\mu} \operatorname{ImI}\right]
$$


where $I$ is the interference term mentioned above, $P_{\mu}$ the beam polarisation and $e_{\mu}$ the beam charge in units of the elementary charge. The distribution of the azimuthal angle $\phi$ between the lepton scattering and the photon production plane is very sensitive to the different contributions.

COMPASS is presently the only facility providing polarised leptons with either charge. Note that with muon beams one naturally reverses both charge and helicity at once. This feature allows with the same apparatus the measurement of the beam charge(C) and $\operatorname{spin}(\mathrm{S})$ sum $\mathscr{S}$ and difference $\mathscr{D}: \mathscr{S}_{C S, U} \equiv d \sigma^{+\downarrow}+d \sigma^{-\uparrow}$ and $\mathscr{D}_{C S, U} \equiv d \sigma^{+\downarrow}-d \sigma^{-\uparrow}$ where the arrow indicates the beam polarisation. From these measurements the real and imaginary part of Compton form factors (CFF) can be extracted. A CFF is a sum over flavours $f$ of convolutions of the respective GPDs with a perturbatively calculable kernel describing the hard $\gamma^{*} q$ interaction.

In the difference $\mathscr{D}_{C S, U}$ the pure BH contribution cancels and the analysis of the $\phi$ dependence will provide a measurements of the real part of the corresponding CFF. The projected accuracy for the $\phi$ dependence of the asymmetry $\mathscr{D}_{C S, U} / \mathscr{S}_{C S, U}$ is shown in Fig.2 left. It is compared to model calculations [8] and the result of a first analysis of the world data [9]. On the other hand in
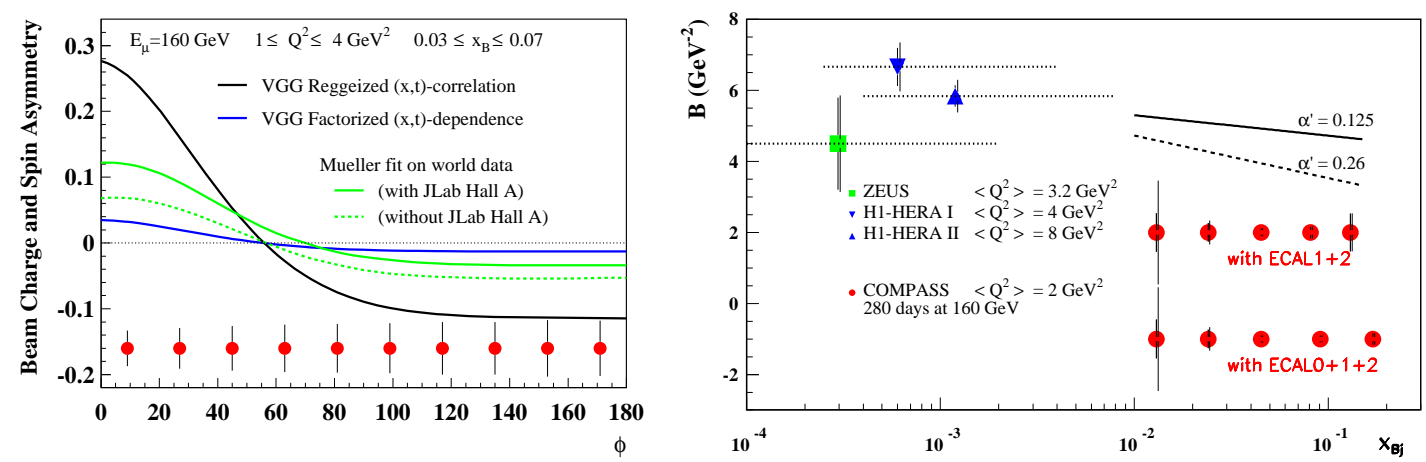

Figure 2: Left: Projected statistical accuracy for a measurement of the $\phi$ dependence of the beam charge and spin asymmetry, for the curves see text. Right: Projections for measuring the $x_{B j}$ dependence of the $t$-slope parameter $B\left(x_{B j}\right)$ of the DVCS cross section compared to previous measurements.

$\mathscr{S}_{C S, U}$ the BH contribution does not cancel and it has to be subtracted provided the contribution is not too large. Integrating over $\phi$ yields the $t$ dependence of the cross section which is related to the transverse size of the nucleon at different values of $x_{B j}$. In the simple ansatz $d \sigma\left(x_{B j}\right) / d t \sim$ $\exp \left(-B\left(x_{B j}\right)|t|\right)$ and $B\left(x_{B j}\right)=B_{0}+2 \alpha^{\prime} \log \left(x_{0} / x_{B j}\right)$ the shrinkage parameter $\alpha^{\prime}$ is used to describe the decrease of the nucleon size with increasing $x_{B j}$. Figure 2 right shows the projected statistical accuracy compared to results from H1 [10] and ZEUS [11] at lower $x_{B j}$.

\section{Test measurements}

For the hadron spectroscopy measurements in 2008 and 2009 with a $40 \mathrm{~cm}$ long liquid hydrogen target a short recoil proton detector used for TOF, energy loss measurements and triggering purposes was constructed. This set-up is an excellent prototype to perform validation measurements for the planned DVCS measurements. Photons were detected in the two existing calorimeters ECAL1 and ECAL2. Using the combined information from the COMPASS spectrometer and the recoil detector an efficient suppression of background is possible. 
A first short measurement of the $\phi$ distribution of real photons was performed in 2008. As expected, the observed distribution for $Q^{2}>1(\mathrm{GeV} / c)^{2}$ is compatible with the dominant $\mathrm{BH}$ process. A detection efficiency of $0.32 \pm 0.13$ was deduced from a comparison with a MC simulation.This motivated a longer test measurement in 2009 where data were taken with $\mu^{+}$and $\mu^{-}$beams. With the increased statistics the $\phi$ dependence was measured in three bins of $x_{B j}$. Figure 3 shows the measured $\phi$ distributions compared to prediction of the $\mathrm{BH}$ process and $\mathrm{BH}+\mathrm{DVCS}$. At low $x_{B j}\left(0.005<x_{B j}<0.01\right) \mathrm{BH}$ is dominant, while at $x_{B j}>0.03$ DVCS is the largest contribution. Note that the predicted $\phi$ dependence of the DVCS contribution is not flat in $\phi$ due to acceptance effects in the 2009 set-up as there is not electromagnetic calorimeter at large photon angles. This will be improved by the addition of ECAL0 as described above.
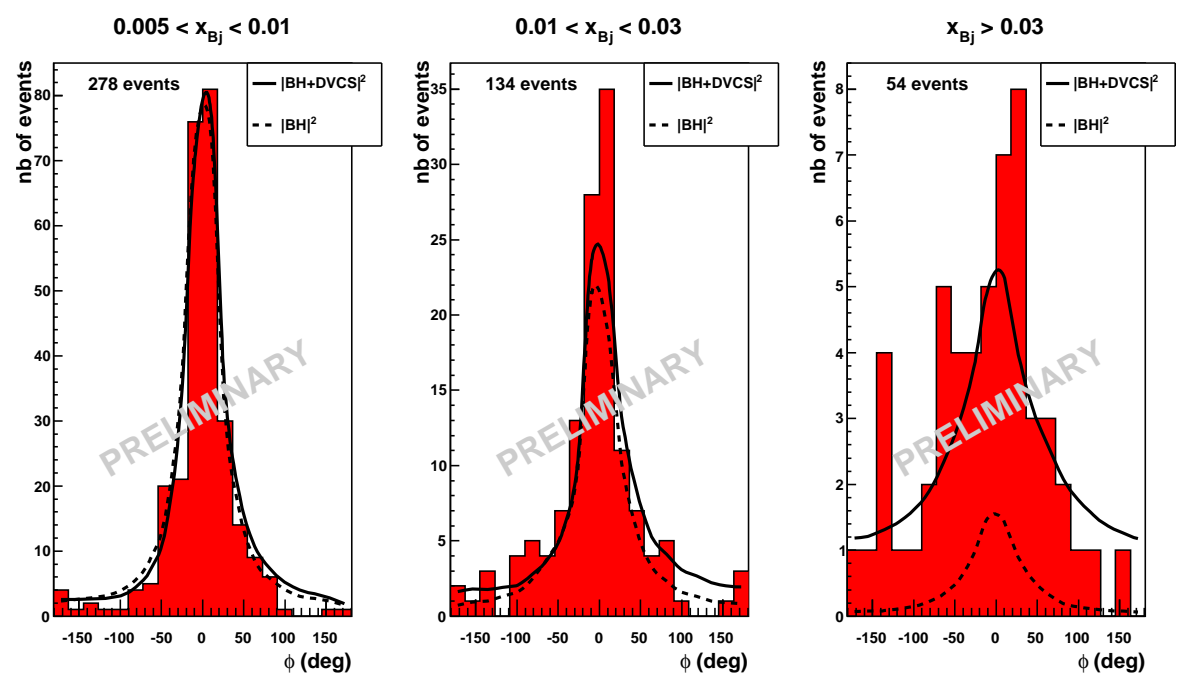

Figure 3: Measured $\phi$ dependence for exclusive single-photon events with $Q^{2}>1(\mathrm{GeV} / c)^{2}$ in three bins of $x_{B j}$ compared to a MC simulation of the BH process only and of both, the BH and DVCS processes.

\section{References}

[1] D. Mueller et al., Fortsch. Phys. 42 (1994) 101.

[2] A.V. Radyushkin, Phys. Lett. B 385 (1996) 333; Phys. Rev. D 56 (1997) 5524.

[3] X. Ji, Phys. Rev. Lett. 78 (1997) 610; Phys. Rev. D 55 (1997) 7114.

[4] M. Burkardt, Phys. Rev. D 62 (2000) 071503, erratum-ibid. D 66 (2002) 119903

[5] COMPASS, SPSC-2010-014/P-340.

[6] COMPASS, P. Abbon et al., Nucl. Instr. Meth. A 577 (2007) 455.

[7] A.V. Belitsky et al., Nucl. Phys. B 629 (2002) 323.

[8] M. Vanderheaghen et al., Phys. Rev. D 60 (1999) 094017.

[9] K. Kumericki, D. Mueller, arXiv 0904.0458[hep-ph].

[10] H1, A. Aktas et al., Eur. Phys. Jour. C 44 (2005)1, F.D. Aaron et al., Phys. Lett. B 659 (2008) 796.

[11] ZEUS, S. Chekanov et al., JHEP 05 (2009) 108. 